\title{
Visualization of and Software for Omnibus Test Based Change Detected in a Time Series of Polarimetric SAR Data
}

Nielsen, Allan Aasbjerg; Conradsen, Knut; Skriver, Henning; Canty, Morton J.

Published in:

Canadian Journal of Remote Sensing

Link to article, DOI:

10.1080/07038992.2017.1394182

Publication date:

2017

Document Version

Peer reviewed version

Link back to DTU Orbit

Citation (APA):

Nielsen, A. A., Conradsen, K., Skriver, H., \& Canty, M. J. (2017). Visualization of and Software for Omnibus Test Based Change Detected in a Time Series of Polarimetric SAR Data. Canadian Journal of Remote Sensing, 43(6), 582-592. https://doi.org/10.1080/07038992.2017.1394182

\section{General rights}

Copyright and moral rights for the publications made accessible in the public portal are retained by the authors and/or other copyright owners and it is a condition of accessing publications that users recognise and abide by the legal requirements associated with these rights.

- Users may download and print one copy of any publication from the public portal for the purpose of private study or research.

- You may not further distribute the material or use it for any profit-making activity or commercial gain

- You may freely distribute the URL identifying the publication in the public portal 


\title{
Visualization of and Software for Omnibus Test Based Change Detected in a Time Series of Polarimetric SAR Data
}

\author{
Allan A. Nielsen, Knut Conradsen, Henning Skriver \& Morton J. Canty
}

To cite this article: Allan A. Nielsen, Knut Conradsen, Henning Skriver \& Morton J. Canty (2017): Visualization of and Software for Omnibus Test Based Change Detected in a Time Series of Polarimetric SAR Data, Canadian Journal of Remote Sensing, DOI: 10.1080/07038992.2017.1394182

To link to this article: https://doi.org/10.1080/07038992.2017.1394182

Accepted author version posted online: 10 Nov 2017.

Submit your article to this journal $[\pi$

山 Article views: 15

Q View related articles $\sqsubset$

View Crossmark data 


\title{
ACCEPTED MANUSCRIPT
}

\section{Visualization of and software for omnibus test based change detected in a time series of polarimetric SAR data}

\author{
Allan A. Nielsen, Knut Conradsen, Henning Skriver, and Morton J. Canty*
}

October 25,2017

\begin{abstract}
Based on an omnibus likelihood ratio test statistic for the equality of several variance-covariance matrices following the complex Wishart distribution and a factorization of this test statistic with associated p-values, change analysis in a time series of multilook polarimetric SAR data in the covariance matrix representation is carried out. The omnibus test statistic and its factorization detect if and when change occurs. Using airborne EMISAR and spaceborne RADARSAT-2 data this paper focuses on change detection based on the p-values, on visualization of change at pixel as well as segment level, and on computer software.

http://www.imm.dtu.dk/pubdb/p.php?6914.
\end{abstract}

Cette étude effectue une analyse sur le changement d'une série temporelle de données multivisées et polarimétriques de radar à synthèse d'ouverture (RSO) représentée sous forme de matrice de covariance. Cette analyse est basée sur 1) un rapport de vraisemblance omnibus pour l'égalité de plusieurs matrices de variance-covariance suivant la distribution de Wishart complexe et 2) une factorisation de celle-ci avec les valeurs-p associées. La statistique de test omnibus et la factorisation correspondante signalent l'occurrence d'un changement. Par moyen de EMISAR aéroportée et RADARSAT-2, cette étude se porte sur la détection de changement en s'appuyant sur les valeurs-p, la visualisation de changement au niveau des pixels et des segments, et des logiciels informatiques.

\section{Introduction}

In the analysis of remote sensing data, a very important aspect is the analysis of spatio-temporal dynamcis including change detection over time. There is an interesting literature on change detection in polarimetric SAR imagery, see for example Dierking, \& Skriver (2002); Qong (2004); Moser, Serpico, \& Vernazza (2007); Moser, \& Serpico (2009); Formont, Pascal, Vasile, Ovarlez, \& Ferro-Famil (2011); Erten, Reigber, \& Ferro-Famil (2012); Marino, Cloude, \& Sanchez-Lopez (2013); Liu, Zhang, Wang, \& Wu (2014); Carotenuto, Maio, Clemente, \& Soraghan (2015); Deledalle, Denis, Tupin, Reigber, \& Jäger (2015); Carotenuto, Maio, Clemente, Soraghan, \& Alfano (2016); Akbari et al. (2016); Yang, Yang, Yan, Song, \& Xia (2016).

In Conradsen, Nielsen, Schou, \& Skriver (2003) we described a likelihood ratio test statistic for equality of two variance-covariance matrices following the complex Wishart distribution and its application to change detection between two time points, see also Canty (2014). We also used this test statistic for edge detection, see Schou, Skriver, Nielsen, \& Conradsen (2003). Special aspects (for example multi-frequency analysis) with computer code were dealt with in Nielsen, Conradsen, \& Skriver (2015).

In Conradsen, Nielsen, \& Skriver (2016) we described a likelihood ratio test statistic $Q$ for equality of a series of variance-covariance matrices following the complex Wishart distribution. We also described a factorization of $Q=\prod_{j=2}^{k} R_{j}$ where the test statistics $Q$ and $R_{j}$ determine if and when a difference occurs. This we used for pixel-wise change detection in a series of multilook polarimetric synthetic aperture radar (SAR) image data in the covariance matrix representation, see van Zyl, \& Ulaby (1990). Additionally, based on approximate distributions of the test statistics we gave $p$-values for $Q$ and $R_{j}$ to determine significance levels for change/no-change.

*A. A. Nielsen and K. Conradsen, DTU Compute, Department of Applied Mathematics and Computer Science, Technical University of Denmark, DK-2800 Kgs. Lyngby, Denmark. H. Skriver, DTU Space, National Space Institute, Technical University of Denmark, DK-2800 Kgs. Lyngby, Denmark. M. J. Canty is an emeritus from the Jülich Research Center, D-52428 Jülich, Germany. Corresponding author: Allan A. Nielsen, e-mail alan@dtu.dk, internet http://people.compute.dtu.dk/alan, phone +4545253425 , fax +45 4588 1397 .

\section{ACC'EPTED MANUSCRIPT}




\section{ACCEPTED MANUSCRIPT}

Generally, one could say that whether you want to characterize a small difference in pixel values as a change event or a random fluctuation, is a philosophical question, it is your choice. In statistically based analysis this choice translates into a choice of significance level.

Because $Q$ tests for equality in all matrices simultaneously, it is termed an omnibus test statistic. Testing all matrices simultaneously makes $Q$ able to detect more subtle, slowly developping changes than the more often used pairwise test statistics.

In this paper we further analyze the change patterns as found by the methods described in Conradsen et al. (2016). Specifically, we give an update scheme to handle the situation where you have analyzed a sequence of images up to time point $j-1$ already and you get new data from time point $j$, we mention/describe Matlab and Python software to perform the analysis, and we give visualization examples of the change detected. This is done in the form of change at field or segment level, and per pixel occurrence of the first and last change as well as the number of changes. Also an RGB representation of per pixel change is given.

Here the methods are demonstrated by means of airborne EMISAR, see Madsen, Christensen, Skou, \& Dall (1991); Christensen et al. (1998) (L-band) data and spaceborne RADARSAT- $2^{1}$ (C-band) data. The methods are well suited for analysis of spaceborne ALOS, COSMO-SkyMed, Sentinel-1, TerraSAR-X, and Yaogan data also.

The following sections very briefly sketch the main results from Conradsen et al. (2016), briefly describe the results output from the analysis, give per pixel and per field change visualization examples, and sketch Matlab and Python code.

\section{Test Statistics and Their Distributions}

This section very briefly recapitulates the main results from Conradsen et al. (2016) on change detection in a series of covariance matrix representation multilook SAR data, see van Zyl, \& Ulaby (1990).

\section{Test for equality of several complex covariance matrices}

For the logarithm of the likelihood ratio test statistic $Q$ for testing whether a series of $k \geq 2$ complex variancecovariance matrices $\boldsymbol{\Sigma}_{i}$ are equal, i.e., $\boldsymbol{\Sigma}_{1}=\boldsymbol{\Sigma}_{2}=\cdots=\boldsymbol{\Sigma}_{k}$ we get (for the real case see Anderson (2003); for the case with two complex matrices see Conradsen et al. (2003); Schou et al. (2003))

$$
\ln Q=n\left\{p k \ln k+\sum_{i=1}^{k} \ln \left|\boldsymbol{X}_{i}\right|-k \ln \left|\sum_{i=1}^{k} \boldsymbol{X}_{i}\right|\right\} .
$$

Here $|\cdot|$ denotes the determinant, $n$ is the equivalent number of looks, the $\boldsymbol{X}_{i}$ (and the $\boldsymbol{\Sigma}_{i}$ ) are $p$ by $p(p=3$ for full pol data, $p=2$ for dual pol data, and $p=1$ for single channel power data), and the $\boldsymbol{X}_{i}=n \hat{\boldsymbol{\Sigma}}_{i}=n\langle\boldsymbol{C}\rangle_{i}$ follow the complex Wishart distribution, i.e., $\boldsymbol{X}_{i} \sim W_{C}\left(p, n, \boldsymbol{\Sigma}_{i}\right)$.

Because $Q$ tests for equality in all matrices simultaneously, it is termed an omnibus test statistic.

In Conradsen et al. (2016) we give $p$-values $1-P\{-2 \rho \ln Q \leq z\}$ for $Q$ to determine significance levels for no-change (where $\rho$ is an auxiliary variable and $z=-2 \rho \ln q_{\text {obs }}$ where $q_{\text {obs }}$ is the observed value of $Q$ ).

\section{Test for equality of first $j \leq k$ matrices}

If the above $\ln Q$ test statistic shows that we cannot reject the hypothesis $\boldsymbol{\Sigma}_{1}=\boldsymbol{\Sigma}_{2}=\cdots=\boldsymbol{\Sigma}_{k}$, there is no change over the time span covered by the data. If we can reject the hypothesis, there is change at some time point. To find that time point, we test whether the first $j(1<j \leq k)$ complex variance-covariance matrices $\boldsymbol{\Sigma}_{i}$ are equal, i.e., given that $\boldsymbol{\Sigma}_{1}=\boldsymbol{\Sigma}_{2}=\cdots=\boldsymbol{\Sigma}_{j-1}$, then the logarithm of the likelihood ratio test statistic $R_{j}$ for testing $\boldsymbol{\Sigma}_{j}=\boldsymbol{\Sigma}_{1}$ is

$$
\begin{aligned}
\ln R_{j}= & n\{p(j \ln j-(j-1) \ln (j-1)) \\
& \left.+(j-1) \ln \left|\sum_{i=1}^{j-1} \boldsymbol{X}_{i}\right|+\ln \left|\boldsymbol{X}_{j}\right|-j \ln \left|\sum_{i=1}^{j} \boldsymbol{X}_{i}\right|\right\} .
\end{aligned}
$$

\footnotetext{
${ }^{1}$ The official RADARSAT-2 web site is http://www.asc-csa.gc.ca/eng/satellites/radarsat2/.
}

\section{ACĆEPTED MANUSCRIPT}




\section{ACCEPTED MANUSCRIPT}

Table 1: Part of the structure wc as output from code wcRjl for an example with data from six time points.

\begin{tabular}{|l|c|c|c|c|c|}
\hline & $t_{1}=\cdots=t_{6}$ & $t_{2}=\cdots=t_{6}$ & $t_{3}=\cdots=t_{6}$ & $t_{4}=\cdots=t_{6}$ & $t_{5}=t_{6}$ \\
\hline Omnibus & $Q^{(1)}:$ wc (1).P(1) & $Q^{(2)}:$ wc (2).P(1) & $Q^{(3)}:$ wc (3).P(1) & $Q^{(4)}:$ wc (4).P(1) & $Q^{(5)}:$ wc (5).P(1) \\
$t_{1}=t_{2}$ & $R_{2}^{(1)}:$ wc(1).P(2) & & & \\
$t_{2}=t_{3}$ & $R_{3}^{(1)}:$ wc(1).P(3) & $R_{2}^{(2)}:$ wc(2).P(2) & & \\
$t_{3}=t_{4}$ & $R_{4}^{(1)}:$ wc(1).P(4) & $R_{3}^{(2)}:$ wc(2).P(3) & $R_{2}^{(3)}:$ wc (3).P(2) & & \\
$t_{4}=t_{5}$ & $R_{5}^{(1)}:$ wc(1).P(5) & $R_{4}^{(2)}:$ wc(2).P(4) & $R_{3}^{(3)}:$ wc(3).P(3) & $R_{2}^{(4)}:$ wc (4).P(2) & \\
$t_{5}=t_{6}$ & $R_{6}^{(1)}:$ wc(1).P(6) & $R_{5}^{(2)}:$ wc(2).P(5) & $R_{4}^{(3)}:$ wc(3).P(4) & $R_{3}^{(4)}:$ wc (4).P(3) & $R_{2}^{(5)}:$ wc (5).P(2) \\
\hline
\end{tabular}

Furthermore, the $R_{j}$ constitute a factorization $Q=\prod_{j=2}^{k} R_{j}$ or

$$
\ln Q=\sum_{j=2}^{k} \ln R_{j} .
$$

In Conradsen et al. (2016) we also gave $p$-values $1-P\left\{-2 \rho_{j} \ln R_{j} \leq z_{j}\right\}$ for $R_{j}$ to determine significance levels for no-change (where $\rho_{j}$ are auxiliary variables and $z_{j}=-2 \rho_{j} \ln r_{j, \text { obs }}$ where $r_{j, \text { obs }}$ are the observed values of $R_{j}$ ).

\section{Update of change detected from time $j-1$ to time $j$}

Equation (2) shows that we can calculate $\ln R_{j}$ based on $\boldsymbol{X}_{j}$ and either all previous data $\boldsymbol{X}_{i}, i=1, \ldots, j-1$ or the sum of all previous data $\sum_{i=1}^{j-1} \boldsymbol{X}_{i}$. To do the former we need to store all previous data, to do the latter we need to store their sum and, since we need the logarithm of the determinant of the sum, preferably also this log-determinant.

\section{$Q$ and $R_{j}$ Based Change Analysis}

This analysis builds a structure of change for each pixel: first we analyze for change over all time points, then for change over all time points omitting the first time point, then for change over all time points omitting the first two time points, etc. The change structure is illustrated in Table 1 for an example with data from six time points. If change is detected comparing for example $t_{2}$ and $t_{3}$ in the " $t_{1}=\cdots=t_{6}$ " column, the remaining tests in that column are invalid and we continue in the column starting with detection of change from $t_{3}$. This will leave the " $t_{2}=\cdots=t_{6} "$ column irrelevant. Continuing like this we can build up the change pattern for all pixels over all time points, see also Conradsen et al. (2016).

Matlab code wcRjl (briefly described in the section on software outputs the structure wc a part of which is shown in Table 1. The first column indicates which tests are performed for the row in question. The second column shows $P\left\{-2 \rho \ln Q^{(1)} \leq z\right\}$ ("Omnibus" row) or $P\left\{-2 \rho_{j} \ln R_{j}^{(1)} \leq z_{j}\right\}, j=2, \ldots, 6$ for all time points $t_{1}$ through $t_{6}$. The third column shows $P\left\{-2 \rho \ln Q^{(2)} \leq z\right\}$ ("Omnibus" row) or $P\left\{-2 \rho_{j} \ln R_{j}^{(2)} \leq z_{j}\right\}, j=2, \ldots, 5$ for time points $t_{2}$ through $t_{6}$. The fourth column shows $P\left\{-2 \rho \ln Q^{(3)} \leq z\right\}$ ("Omnibus" row) or $P\left\{-2 \rho_{j} \ln R_{j}^{(3)} \leq z_{j}\right\}, j=2, \ldots, 4$ for time points $t_{3}$ through $t_{6}$, etc. For a test for $R_{j}^{(\ell)}$ to be valid, all previous tests for $R_{i}^{(\ell)}, i=2, \ldots, j-1$ must show equality.

$R_{2}^{(\ell)}, \ell=1, \ldots, k-1$ is the pairwise (or marginal or "non-omnibus") test statistics for equality between consecutive time points $t_{\ell}$ and $t_{\ell+1}$ (as described in Conradsen et al. (2003)). Note, that for the last column $Q=R_{2}$.

$P\left\{-2 \rho \ln Q^{(\ell)} \leq z\right\}$ is stored in wc $(\ell) . P(1)$, and $P\left\{-2 \rho_{j} \ln R_{j}^{(\ell)} \leq z_{j}\right\}$ is stored in wc $(\ell) . P(j)$.

\section{Change Visualization Examples}

To illustrate results from analyses by means of the Matlab code mentioned in the sction on software we use full polarimetry EMISAR L-band data acquired in 1998 over a Danish agricultural test site on $t_{1}=21$ March $($ dataset

\section{ACČ EPTED MANUSCRIPT}




\section{ACCEPTED MANUSCRIPT}

L62), $t_{2}=17$ April (dataset L63), $t_{3}=20$ May (dataset L64), $t_{4}=16$ June (dataset L65), $t_{5}=15$ July (dataset L68), and $t_{6}=16$ August (dataset L74). The 13-look images are 1024 rows by 1024 columns $5 \mathrm{~m}$ pixels, see Conradsen et al. (2016) for more details.

To generate the desired change structure call

$\mathrm{wC}=\mathrm{wCRj} 1(\operatorname{cat}(4, \mathrm{~L} 62, \mathrm{~L} 63, \mathrm{~L} 64, \mathrm{~L} 65, \mathrm{~L} 68, \ldots$

L74), 13, 'full', false);

for a structure without storing the pixelwise sum of all data and the logarithm of the determinant of the sum (false in the call). Alternatively, to store these quantities call either (you need not specify the polarization)

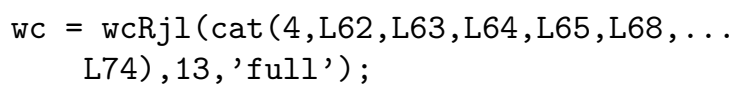

or for example (again the pol specification is not needed)

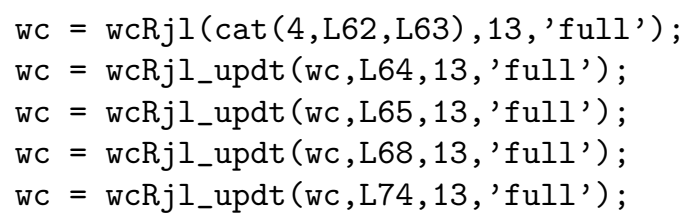

\section{Per pixel change visualization}

Given the structure wc as output from wcRjl or wcRjl_updt (we need wc.P only) and a threshold for the change probability, the Matlab function wcWhen finds when change(s) occurred on a pixel basis. Following the call

$[\mathrm{c}, \mathrm{nsets}]=$ wcWhen $(\mathrm{wc}, 0.9999) ;$

this code finds when the first change occurred at a change probability significance level of $99.99 \%$ (corresponding to a no-change probability significance level of $0.01 \%$ )

[maxch, changeFirst $]=\max (c,[], 3)$;

changeFirst $(\operatorname{maxch}==0)=0$;

For the six L-band EMISAR data sets also analyzed in Conradsen et al. (2016) this code

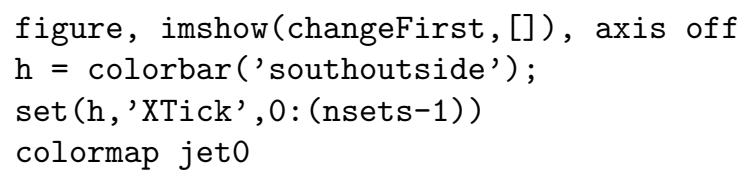

generates Figure 1 which shows the time point at which the first detected change took place for full polarization data (change probability significance level is $99.99 \%$ ). "jet0" is Matlab's "jet" colormap with the first entry set to black. Since we expect to analyze also very long time series for which a continuous colour scale is to be preferred, we choose to use that colour scale here also with six time points only.

This code finds when the last/most recent change occurred at a change probability significance level of $99.99 \%$ (corresponding to a no-change probability significance level of $0.01 \%$ ) and generates Figure 2

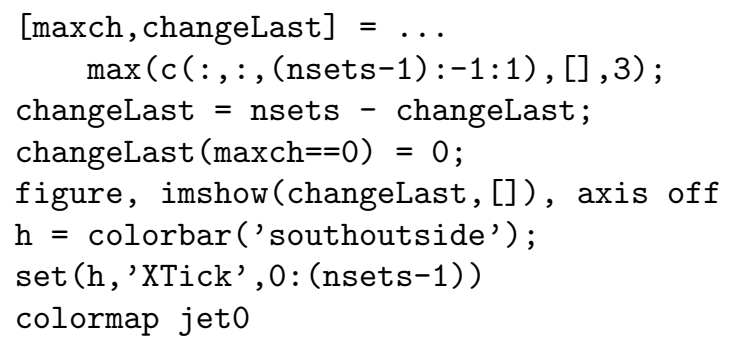

This code finds the number of changes/change frequency and generates Figure 3

\section{ACĆEPTED MANUSCRIPT}




\section{ACCEPTED MANUSCRIPT}

Table 2: Average no-change probabilities for a grass field.

\begin{tabular}{|r|r|r|r|r|r|}
\hline & $t_{1}=\cdots=t_{6}$ & $t_{2}=\cdots=t_{6}$ & $t_{3}=\cdots=t_{6}$ & $t_{4}=\cdots=t_{6}$ & $t_{5}=t_{6}$ \\
\hline Omnibus & 0.0000 & 0.0000 & 0.0000 & 0.0000 & 0.0000 \\
$t_{1}=t_{2}$ & 0.2808 & & & & \\
$t_{2}=t_{3}$ & 0.0112 & 0.0638 & & & \\
$t_{3}=t_{4}$ & 0.0056 & 0.0319 & 0.1244 & & \\
$t_{4}=t_{5}$ & 0.1094 & 0.2386 & 0.3879 & 0.4311 & \\
$t_{5}=t_{6}$ & 0.0000 & 0.0000 & 0.0000 & 0.0000 & 0.0000 \\
\hline
\end{tabular}

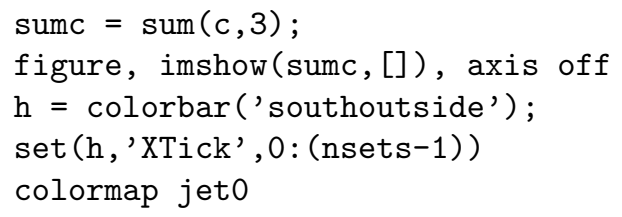

As a further example of per pixel change visualization of the same data, this generates Figure 4

figure, imshow(single $\left.\left(c\left(:,:,\left[\begin{array}{lll}1 & 3 & 5\end{array}\right]\right)\right)\right)$

axis off

which shows changes from $t_{1}$ to $t_{2}$ (in the beginning of the time series) as red, from $t_{3}$ to $t_{4}$ (in the middle of the time series) as green, and from $t_{5}$ to $t_{6}$ (in the end of the time series) as blue. This (of course) means that for example yellow areas have changed during time intervals $t_{1}-t_{2}$ and $t_{3}-t_{4}$, white areas have changed during time intervals $t_{1}-t_{2}, t_{3}-t_{4}$, and $t_{5}-t_{6}$. Black areas have not changed.

Finally, for illustration the Python scripts were used to perform change analysis of a series of 12 quad polarimetric RADARSAT-2 images acquired near the city of Bonn, Germany between May, 2009 and October, 2010. Figure 7 shows a $400 \times 400$-pixel spatial subset of the first image in the series. The dark feature near the center is a flooded sand quarry. The corresponding change frequency map is shown in Figure 8, where there is seen to be a "hot spot" in the sand quarry caused by the continual movement of two dredging arms.

\section{Per field change visualization}

Table 2 shows the average no-change probabilities for a field (the field chosen is the grass field also analyzed in Conradsen et al. (2016); it is chosen because the omnibus test statistic detects change not detected by the pairwise test statistics). Figure 5 shows the same probabilities as graphs, one line per column in Table 2. The omnibus test based no-change probabilities shown along with the pairwise no-change probabilities in the bottom plot in Figure 6 are highlighted in yellow. These numbers show that at a $5 \%$ significance level the pairwise tests show no change over the time span March to July. Contrary to this the omnibus tests show change between April and May (and also between July and August). If we apply a $1 \%$ significance level change is detected between May and June, and between July and August.

As an example of visualization of per field change detection Figure 6 shows mean values in the grass field of the backscatter coefficients and the span (first plot), the determinant of the covariance matrix multiplied by the equivalent number of looks (second plot), the magnitude of the correlation between HH and VV (third plot), and the phase difference between $\mathrm{HH}$ and $\mathrm{VV}$ for all six acquisitions (fourth plot). The last plot shows the average pairwise and omnibus based no-change probabilities (at a $5 \%$ level).

The parameters in Figure 6 can be interpreted in terms of the scattering mechanisms for the grass vegetation. In March and April, the low HV backscatter, the larger VV than HH backscatter, the relatively high correlation coefficient, and some phase difference between $\mathrm{HH}$ and VV indicate that the scattering is dominated by surface scattering and some double bounce scattering from the not so dense vegetation. In May, June and July, the higher HV backscatter, the same $\mathrm{HH}$ and VV backscatter, the lower correlation coefficient, and a phase difference close to zero indicate volume scattering from the dense vegetation. In August, where the grass has been cut, the parameters

\section{ACĆEPTED MANUSCRIPT}




\section{ACCEPTED MANUSCRIPT}

indicate surface scattering, i.e., low HV backscatter, larger VV than HH backscatter, correlation coefficient close to one, and phase difference close to zero. This interpretation matches clearly with the three no-change segments at the $5 \%$ significance level indicated above: 1) March and April, 2) May, June and July, and 3) August.

We expect that plots like these will be even more useful for interpreting change in longer time series where possible periodical, step and impulse changes will be conspicuous.

Finally, we show an example of visualization of per field change detection for the RADARSAT example. Figure 9 shows the sample and theoretical distributions of $-2 \rho \ln Q$ and $-2 \rho_{j} \ln R_{j}$ for a no-change wooded region. Judged visually these compare satisfactorily.

The visualization examples shown in this subsection are field or patch based. However the actual change detection is pixel based. The change detection itself could be patch based also. Following some segmentation of the imagery, after adjustment for the number of looks which will depend on the segment size, the change detection method sketched above could be applied to the segments. This is not pursued further here.

For no-change examples, see the wooded areas in the pixelswise visualizations (most of the black areas in Figures 1, $2,3,4$, and 8 ).

\section{Software}

Both Matlab and Python code to carry out the analyses described are available.

\section{Matlab Code}

Several Matlab functions are available on Allan Nielsen's homepage ${ }^{2}$

- freadenvisar,

- rho_hhvv,

- wishart_det,

- wishart_change_Q,

- wishart_change $R j$,

- wcRjl,

- wishart_change_Rj_updt,

- wcRj1_updt,

- wcWhen,

- wcPtable,

- wclnRtable.

freadenvisar reads covariance matrix polarimetric SAR data (with an ENVI-like header file) from disk and stores them in the order needed for the change detection programs. You may need to change the file names in the code. Also some files are written in row major order and some in column major order, so there may be an issue with transposing the images read.

rho_hhvv calculates the complex correlation between HH and VV polarizations (including the phase difference) for full polarimetry, azimuthal symmetry, and dual polarimetry with $\mathrm{HH}$ and VV.

wishart_det calculates determinants of complex variance-covariance matrices and flags singularity for full polarimetry (including azimuthal symmetry and diagonal only), and dual polarimetry (including diagonal only).

wishart_change_Q calculates $\ln Q$ and the $p$-value to test for equality of $k \geq 2$ covariance matrices in the full and dual polarimetry cases and of single band power data (any polarization). wishart_change_Q calculates $\ln Q$ as in (1).

wishart_change_Rj calculates $\ln Q, \ln R_{j}$ and the associated $p$-values to test for equality of covariance matrices in full and dual polarimetry data as well as in single band power data, $k \geq 2$. wishart_change $\operatorname{Rj}$ calculates $\ln Q$ as

\footnotetext{
${ }^{2}$ http://people.compute.dtu.dk/alan/ under Software
} 


\section{ACCEPTED MANUSCRIPT}

in (3). This code also exists in a version which reads, processes and writes data from/to disk line-by-line making it useful for large datasets that do not fit into computer memory.

wcRjl is a wrapper which calls wishart_change $R j$ several times, first with data from all time points (which generates output for the second column of Table 1), then with data from all time points omitting the first, (which generates output for the third column of Table 1), then with data from all time points omitting the two first (which generates output for the fourth column of Table 1), etc. The output is a structure wc which contains

- wc $(\ell) \cdot \ln R\left(\ln Q\right.$ and $\ln R_{j} ; \ln Q$ is stored in $\operatorname{lnR}(:,:, 1), \ln R_{2}$ in $\operatorname{lnR}(:,:, 2)$, etc.),

- $\operatorname{wc}(\ell) . f(f)$,

- wc $(\ell)$.rho $\left(\rho\right.$ and $\left.\rho_{j}\right)$,

- $\operatorname{wc}(\ell)$. omega2 $\left(\omega_{2}\right.$ and $\left.\omega_{2 j}\right)$, and

- wc $(\ell)$.P ( $p$-values, stored in the same fashion as $\ln Q$ and $\left.\ln R_{j}\right)$.

For $\ell=1$ all time points are considered, for $\ell=2$ all time points omitting the first are considered, for $\ell=3$ all time points omitting the two first are considered, etc. The structure may optionally contain

- wc $(\ell)$. sumMat (the $\operatorname{sum} \sum_{i=1}^{j} \boldsymbol{X}_{i}$ ) and

- wc $(\ell)$. logDet (the logarithm of the determinant of this sum mentioned in the section on updating of change).

For large data sets the structure wc takes up a lot of computer memory. You may want to output wc.P only. Also you may want to store this in four as opposed to eight byte precision.

wishart_change_Rj_updt: given data from $k$ time points wishart_change_Rj_updt based on new data in $\boldsymbol{X}_{k+1}$, calculates $\ln R_{k+1}, p$-values and auxiliary variables ( $f$ etc.) for full and dual polarimetry data as well as for single band power data.

wcRjl_updt like wcRjl is a wrapper. wcRjl_updt calls wishart_change_Rj_updt several times, first with either data from all time points or the sum of data from all time points (which updates output for the second column of Table 1 with an extra row followed by an update of $\ln Q$ based on Equation (3)), then with either data from all time points or the sum of data from all time points, omitting the first, (which updates output for the third column of Table 1 with an extra row followed by an update of $\ln Q$ based on Equation (3)), then with either data from all time points or the sum of data from all time points, omitting the two first (which updates output for the fourth column of Table 1 with an extra row followed by an update of $\ln Q$ based on Equation (3)), etc. A version called wcRj1_data_updt that handles the situation with no storage of the sum $\sum_{i=1}^{j} \boldsymbol{X}_{i}$ (and the logarithm of the determinant of this sum) mentioned in the section on updating is also available.

wcWhen examines the change probabilities $(P)$ in the structure wc output by wcRjl and outputs all occurrences of change (given a change probability significance level).

wcPtable calculates the no-change probabilities $(1-P)$ from the structure wc output by wcRjl for a region of interest (ROI) and outputs a table such as Table 1 (or Table 2). It also plots histograms for the no-change probabilities in the ROI. These histograms are flat for a ROI with no change (the distribution of $1-P$ is uniform). You may input a ROI image or a colour image on which to draw a ROI polygon.

wclnRtable calculates $-2 \rho \ln Q$ and $-2 \rho_{j} \ln R_{j}$ from the structure wc output by wcRjl for a region of interest (ROI) and outputs a table such as Table 1. It also plots sample histograms and theoretical distributions for the these quantities in the ROI. Figure 9 shows that judged visually these two match satisfactorily for a no-change region.

\section{Python Code}

Python scripts for ( $Q$ and) $R_{j}$ based change detection are available at Docker Hub ${ }^{3}$. The software package, a so-called Docker image, can be pulled and run as a Docker container from any host computer which has the Docker Engine installed, freely available for Linux, Windows and Mac $\mathrm{OS}^{4}$. In addition to a complete Linux environment and all necessary Python prerequisites and scripts for polarimetric SAR change detection, the Docker container encapsulates Python code for speckle filtering and for multivariate estimation of the equivalent number of looks (ENL) of an image in polarimetric matrix format Anfinsen, Doulgeris, \& Eltoft (2009). The container also includes the command line interface of the ASF MapReady software for terrain correction and geocoding of SAR images ${ }^{5}$. The user interacts

\footnotetext{
${ }^{3}$ https://hub.docker.com/u/mort/

${ }^{4}$ https://docs.docker.com/

${ }^{5}$ https://www.asf.alaska.edu/data-tools/mapready/
} 


\section{ACCEPTED MANUSCRIPT}

with the software in his or her browser in an IPython notebook served from within the Docker container. Parallel computation on multi-core processors is possible if the user enables the corresponding number of IPython engines. The change detection script is sar_seq.py. This script accepts as input a comma-separated list of filenames for the multi-temporal polarimetric image sequence. If required, the images are co-registered to the first image in the sequence and clipped to the same spatial dimensions. Then the per-pixel $R_{j}$ values for the entire sequence are pre-calculated and their $p$-values are stored in a memory-mapped array on disk. Both of these steps are run in parallel on IPython engines if available, otherwise they are performed sequentially. Finally the $p$-value array is scanned at the desired significance level, and four byte arrays having the spatial dimensions of the images are updated recursively in order to generate geo-referenced change maps. These are:

- c_map: the interval in which the most recent significant change occurred (single-band),

- s_map: the interval in which the first significant change occurred (single-band),

- f_map: the frequency of significant changes (single-band), and

- b_map: the interval in which each significant change occurred $((k-1)$-band).

A typical output log is shown below for a sequence of 12 RADARSAT-2 quad polarimetric images. The calculation was performed on an Intel quad core $1.7 \mathrm{GHz}$ processor with four IPython engines enabled.

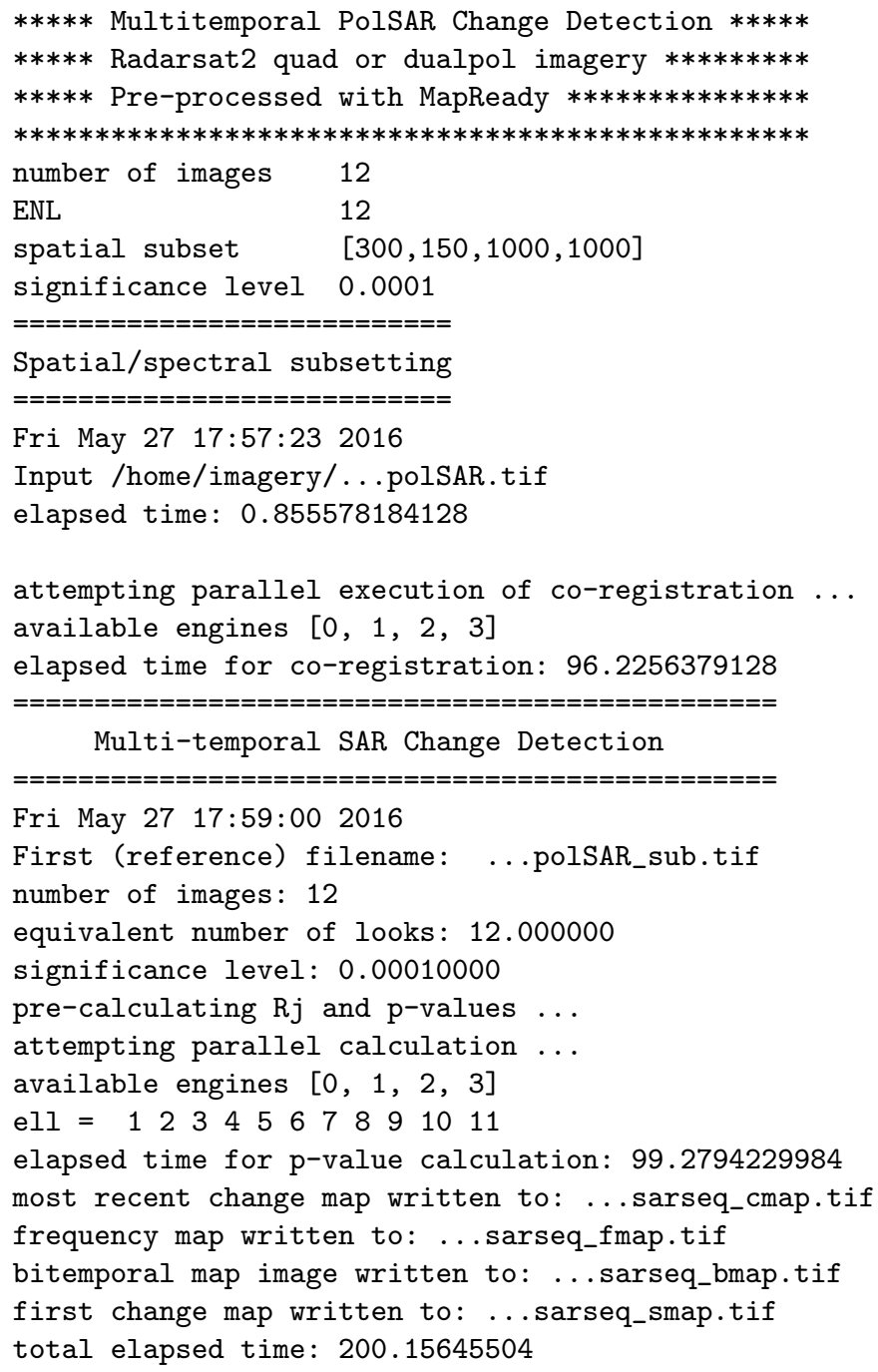




\section{ACCEPTED MANUSCRIPT}

The complete source code for the Docker image is available on GitHub ${ }^{6}$ under the MIT license. Also a tutorial notebook in HTML format can be found on Github ${ }^{7}$.

\section{Conclusions}

Based on the omnibus test statistic and its factorization for change detection in truly multitemporal, multilook, polarimetric SAR data described in Conradsen et al. (2016), we briefly describe software to perform the analysis, we give per pixel change visualization examples, specifically the time point of the first change, the time point of the last/most recent change, the number of changes/change frequency, and we give an example of an RGB representation of change in the beginning, the middle and the end of the time series analyzed. Also, a per field visualization example which is meant to assist an analyst in determining the type of change is given.

Here, for the grass field selected the pairwise tests showed change at a 5\% level between July and August only, whereas the omnibus tests showed change between April and May as well as between July and August. If we choose a $1 \%$ level, the change detected between April and May is replaced by change between May and June.

Also an example on omnibus change detection based on a series of quad polarization RADARSAT-2 images is shown. This example shows a "hot spot" in a sand quarry caused by the continual movement of two dredging arms. It also gives an example on the theoretical and sample distributions of the test statistics in a (wooded) no-change region. The two compare satisfactorily.

The accompanying software is expected to be useful to both researchers and practioners working on time series of polarimetric SAR data.

\section{References}

Akbari, V., Anfinsen, S. N., Doulgeris, A. P., Eltoft, T., Moser, G., \& Serpico, S. B. (2016, Jul.). Polarimetric SAR change detection with the complex Hotelling-Lawley trace statistic. IEEE Transactions on Geoscience and Remote Sensing, 54(7), 3953-3966.

Anderson, T. W. (2003). An introduction to multivariate statistical analysis (Third ed.). John Wiley, New York.

Anfinsen, S. N., Doulgeris, A. P., \& Eltoft, T. (2009, Nov.). Estimation of the equivalent number of looks in polarimetric synthetic aperture radar imagery. IEEE Transactions on Geoscience and Remote Sensing, 47(11), 3795-3809.

Canty, M. J. (2014). Image analysis, classification and change detection in remote sensing: With algorithms for ENVI/IDL and Python (Third ed.). Taylor \& Francis, CRC Press.

Carotenuto, V., Maio, A. D., Clemente, C., \& Soraghan, J. J. (2015, Jun.). Invariant rules for multipolarization sar change detection. IEEE Transactions on Geoscience and Remote Sensing, 53(6), 3294-3311.

Carotenuto, V., Maio, A. D., Clemente, C., Soraghan, J. J., \& Alfano, G. (2016, Jan.). Forcing scale invariance in multipolarization sar change detection. IEEE Transactions on Geoscience and Remote Sensing, 54(1), 36-50.

Christensen, E. L., Skou, N., Dall, J., Woelders, K., Jørgensen, J. H., Granholm, J., \& Madsen, S. N. (1998). EMISAR: An absolutely calibrated polarimetric L- and C-band SAR. IEEE Transactions on Geoscience and Remote Sensing, 36, 1852-1865.

Conradsen, K., Nielsen, A. A., Schou, J., \& Skriver, H. (2003, Jan.). A test statistic in the complex Wishart distribution and its application to change detection in polarimetric SAR data. IEEE Transactions on Geoscience and Remote Sensing, 41(1), 4-19. Retrieved from http://ieeexplore.ieee.org/Xplore/ RecentIssue.jsp?Punumber=36 (http://www.imm.dtu.dk/pubdb/p.php?1219)

Conradsen, K., Nielsen, A. A., \& Skriver, H. (2016, May). Determining the points of change in time series of polarimetric SAR data. IEEE Transactions on Geoscience and Remote Sensing, 54(5), 3007-3024. (http://www.imm.dtu.dk/pubdb/p.php?6825)

Deledalle, C. A., Denis, L., Tupin, F., Reigber, A., \& Jäger, M. (2015). NL-SAR: A unified nonlocal framework for resolution-preserving (Pol)(In) SAR denoising. IEEE Transactions on Geoscience and Remote Sensing, 53(4), 2021-2038.

Dierking, W., \& Skriver, H. (2002, Mar.). Change detection for thematic mapping by means of airborne multitemporal polarimetric SAR imagery. IEEE Transactions on Geoscience and Remote Sensing, 40(3), 618-636.

\footnotetext{
${ }^{6}$ http://mortcanty.github.io/SARDocker/

${ }^{7}$ http://mortcanty.github.io/src/tutorialsar.html
}

\section{ACCُEPTED MANUSCRIPT}




\section{ACCEPTED MANUSCRIPT}

Erten, E., Reigber, A., \& Ferro-Famil, L. (2012, Dec.). A new coherent similarity measure for temporal multichannel scene characterization. IEEE Transactions on Geoscience and Remote Sensing, 52(12), 2839-2851.

Formont, P., Pascal, F., Vasile, G., Ovarlez, J. P., \& Ferro-Famil, L. (2011, Jun.). Statistical classification for heterogeneous polarimetric SAR images. IEEE Journal of Selected Topics in Signal Processing, 5(3), 567-576.

Liu, M., Zhang, H., Wang, C., \& Wu, F. (2014, Jul.). Change detection of multilook polarimetric SAR images using heterogeneous clutter models. IEEE Transactions on Geoscience and Remote Sensing, 50(7), 7483-7494.

Madsen, S. N., Christensen, E. L., Skou, N., \& Dall, J. (1991). The Danish SAR system: Design and initial tests. IEEE Transactions on Geoscience and Remote Sensing, 29, 417-426.

Marino, A., Cloude, S., \& Sanchez-Lopez, J. (2013, May). A new polarimetric change detector in radar imagery. IEEE Transactions on Geoscience and Remote Sensing, 51(5), 2986-3000.

Moser, G., \& Serpico, S. B. (2009, Jul.). Unsupervised change detection from multichannel SAR data by Markovian data fusion. IEEE Transactions on Geoscience and Remote Sensing, 47(7), 2114-2128.

Moser, G., Serpico, S. B., \& Vernazza, G. (2007, Apr.). Unsupervised change detection from multichannel SAR images. IEEE Geoscience and Remote Sensing Letters, 4(2), 278-282.

Nielsen, A. A., Conradsen, K., \& Skriver, H. (2015, Aug.). Change detection in full and dual polarization, single- and multi-frequency SAR data. IEEE Journal of Selected Topics in Applied Earth Observations and Remote Sensing, 8(8), 4041-4048. (http://www.imm.dtu.dk/pubdb/p.php?6827)

Qong, M. (2004, Oct.). Polarization state conformation and its application to change detection in polarimetric SAR data. IEEE Geoscience and Remote Sensing Letters, 1(4), 304-307.

Schou, J., Skriver, H., Nielsen, A. A., \& Conradsen, K. (2003, Jan.). CFAR edge detector for polarimetric SAR images. IEEE Transactions on Geoscience and Remote Sensing, 41(1), 20-32. http://ieeexplore.ieee.org (http://www.imm.dtu.dk/pubdb/p.php?1224)

van Zyl, J. J., \& Ulaby, F. T. (1990). Scattering matrix representation for simple targets. In F. T. Ulaby \& C. Elachi (Eds.), Radar polarimetry for geoscience applications. Artech, Norwood, MA.

Yang, W., Yang, X., Yan, T., Song, H., \& Xia, G.-S. (2016). Region-based change detection for polarimetric SAR images using Wishart mixture models. IEEE Transactions on Geoscience and Remote Sensing, 54(11), 67466756. (https://doi.org/10.1109/TGRS.2016.2590145)

10 


\section{ACCEPTED MANUSCRIPT}

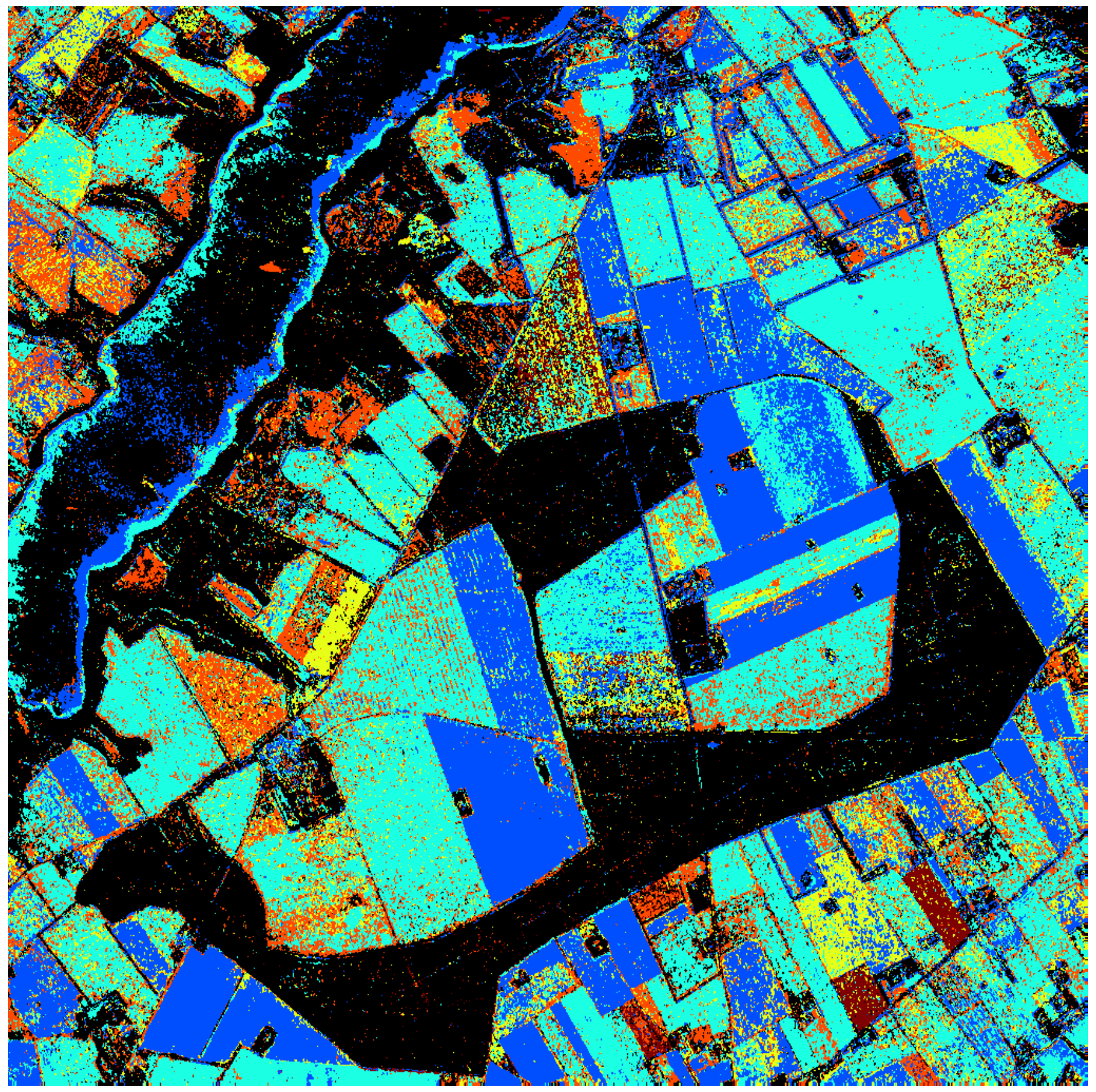

\section{0}

1

2

3

4

Figure 1: Shows when the first detected change occurred, zero (black) means no change over the time span, one means first change occurred between $t_{1}$ and $t_{2}$, etc. No change occurred in wooded regions and most of the lake to the north-west. Change probability significance level (for full polarization data) is $99.99 \%$. 


\section{ACCEPTED MANUSCRIPT}
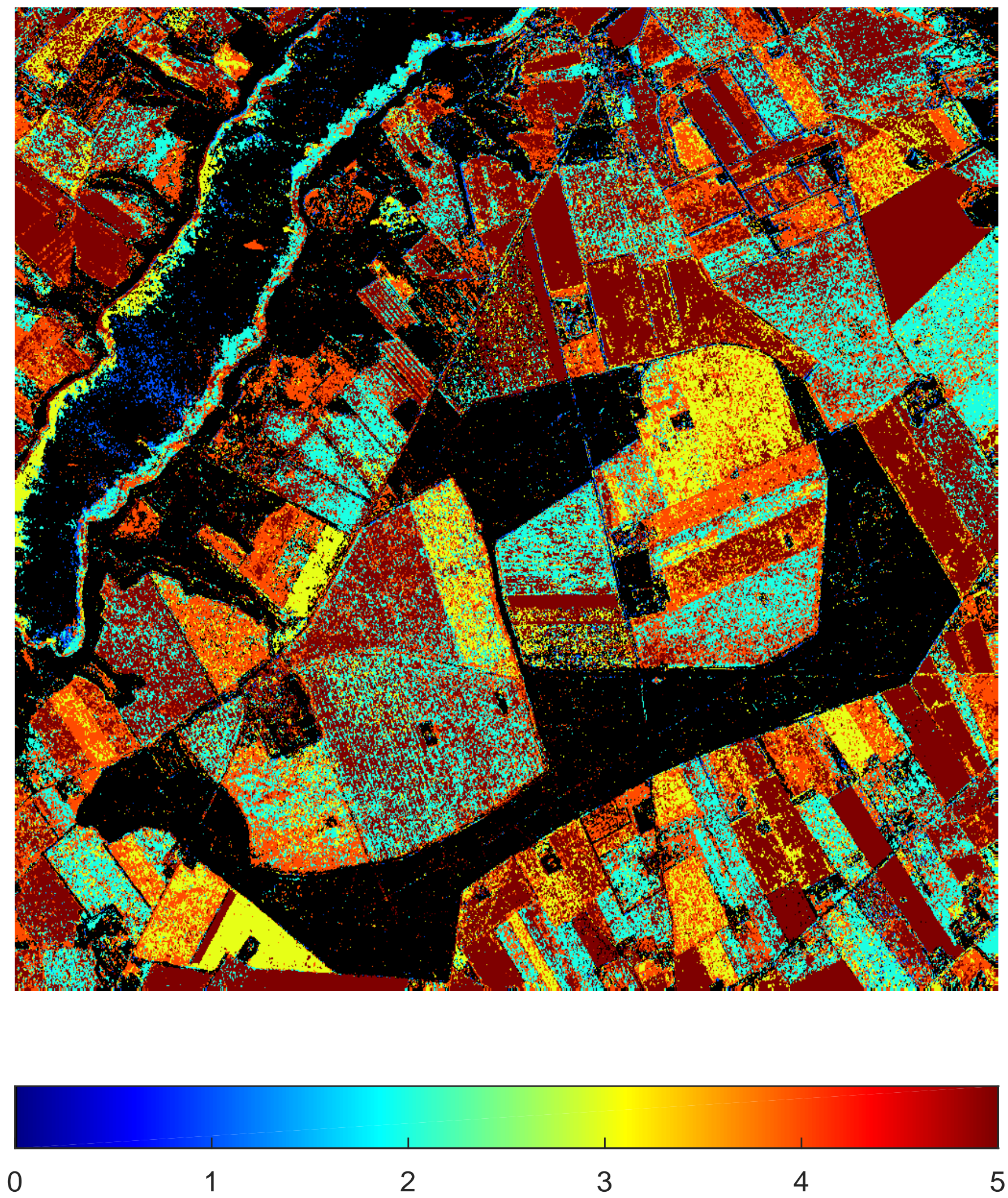

Figure 2: Shows when the last /most recently detected change occurred, zero (black) means no change over the time span, one means first change occurred between $t_{1}$ and $t_{2}$, etc. Change probability significance level (for full polarization data) is $99.99 \%$. 


\section{ACCEPTED MANUSCRIPT}
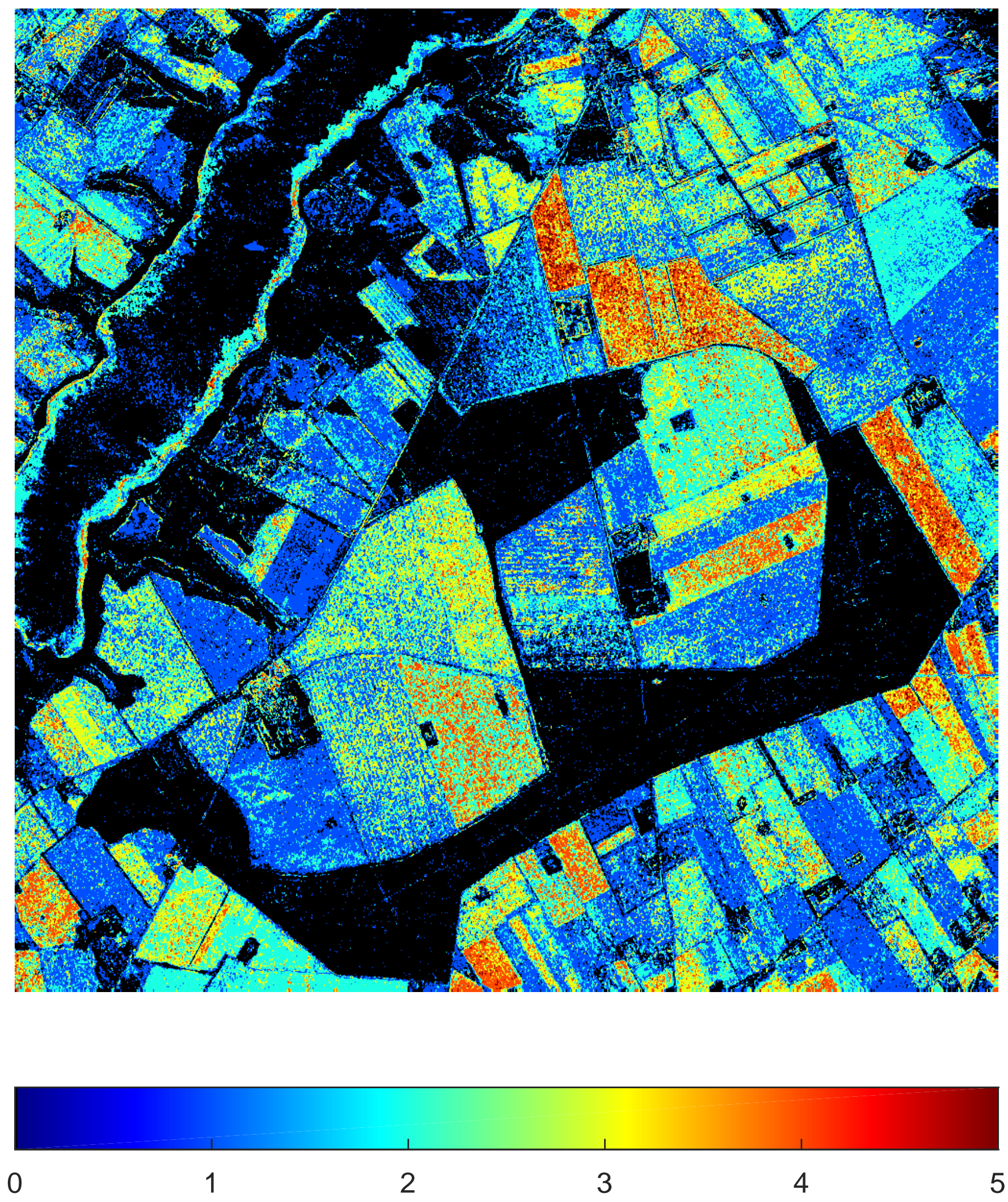

Figure 3: Shows the number of changes/change frequency, again zero (black) means no change. Change probability significance level (for full polarization data) is $99.99 \%$. 


\section{ACCEPTED MANUSCRIPT}

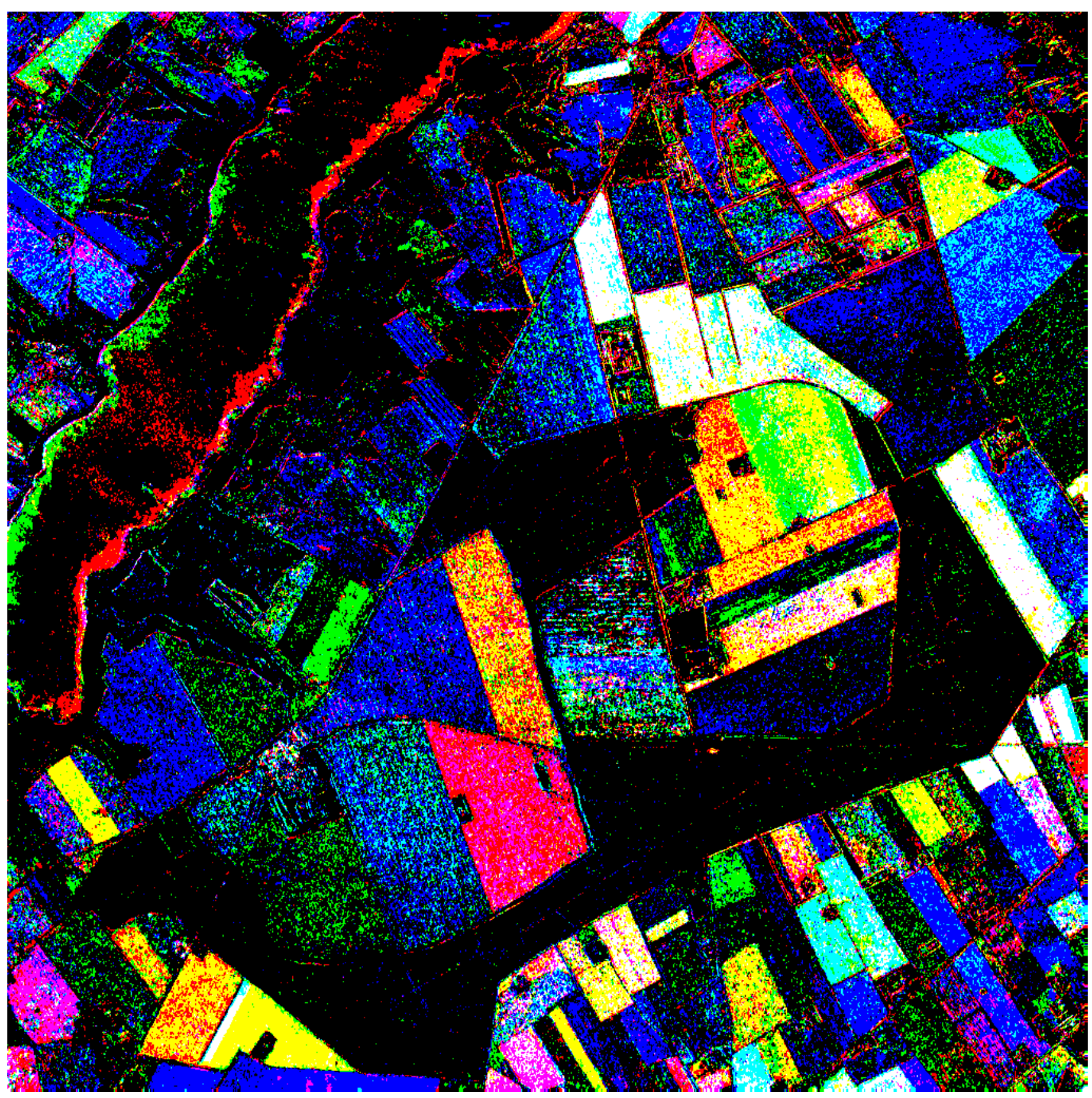

Figure 4: Shows changes from $t_{1}$ to $t_{2}$ as red, from $t_{3}$ to $t_{4}$ as green, from $t_{5}$ to $t_{6}$ as blue (black means no change in the time intervals depicted). Change probability significance level (for full polarization data) is $99.99 \%$. 


\section{ACCEPTED MANUSCRIPT}

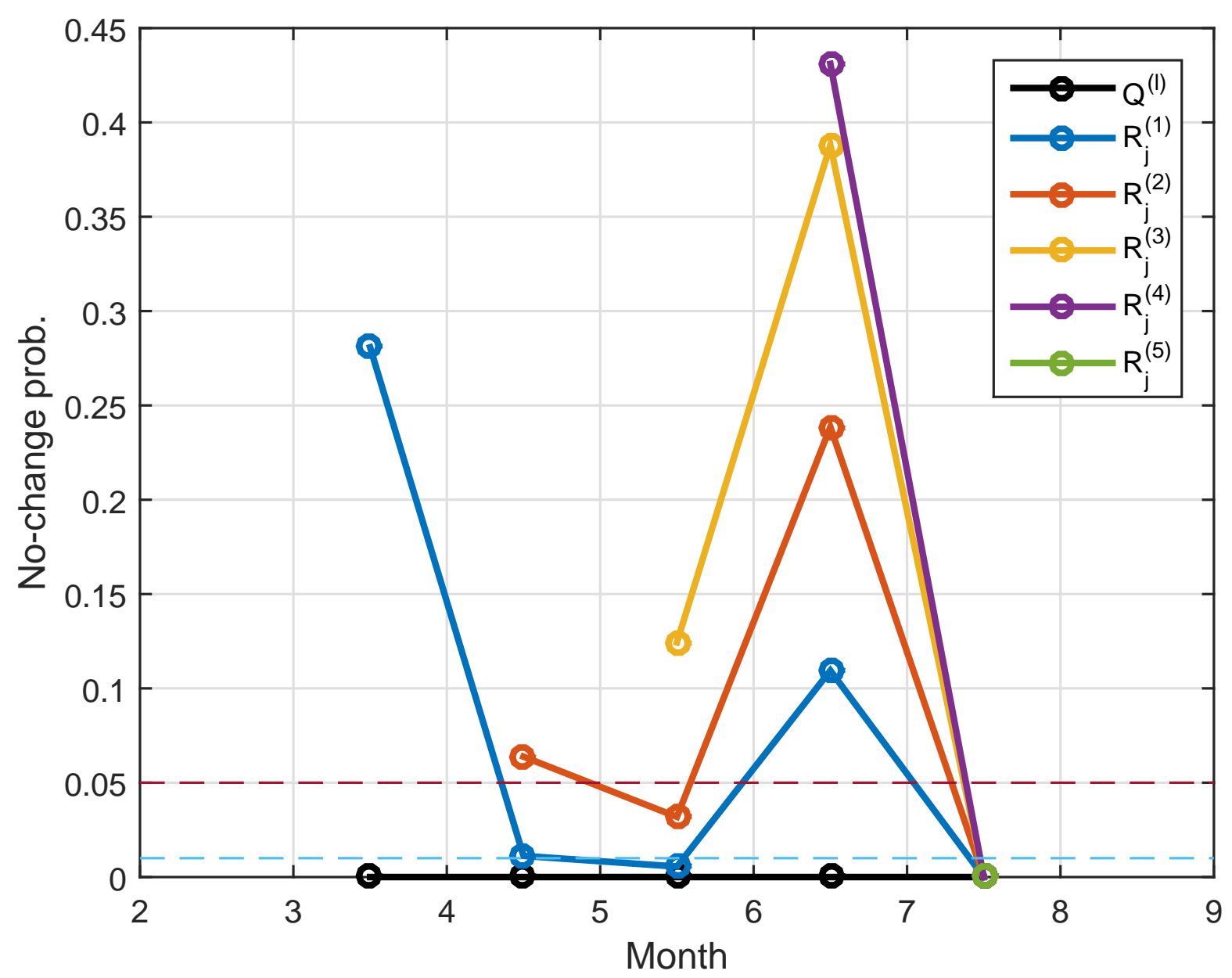

Figure 5: For a grass field this figure shows average omnibus test based no-change probabilities tabulated in Table 2. 

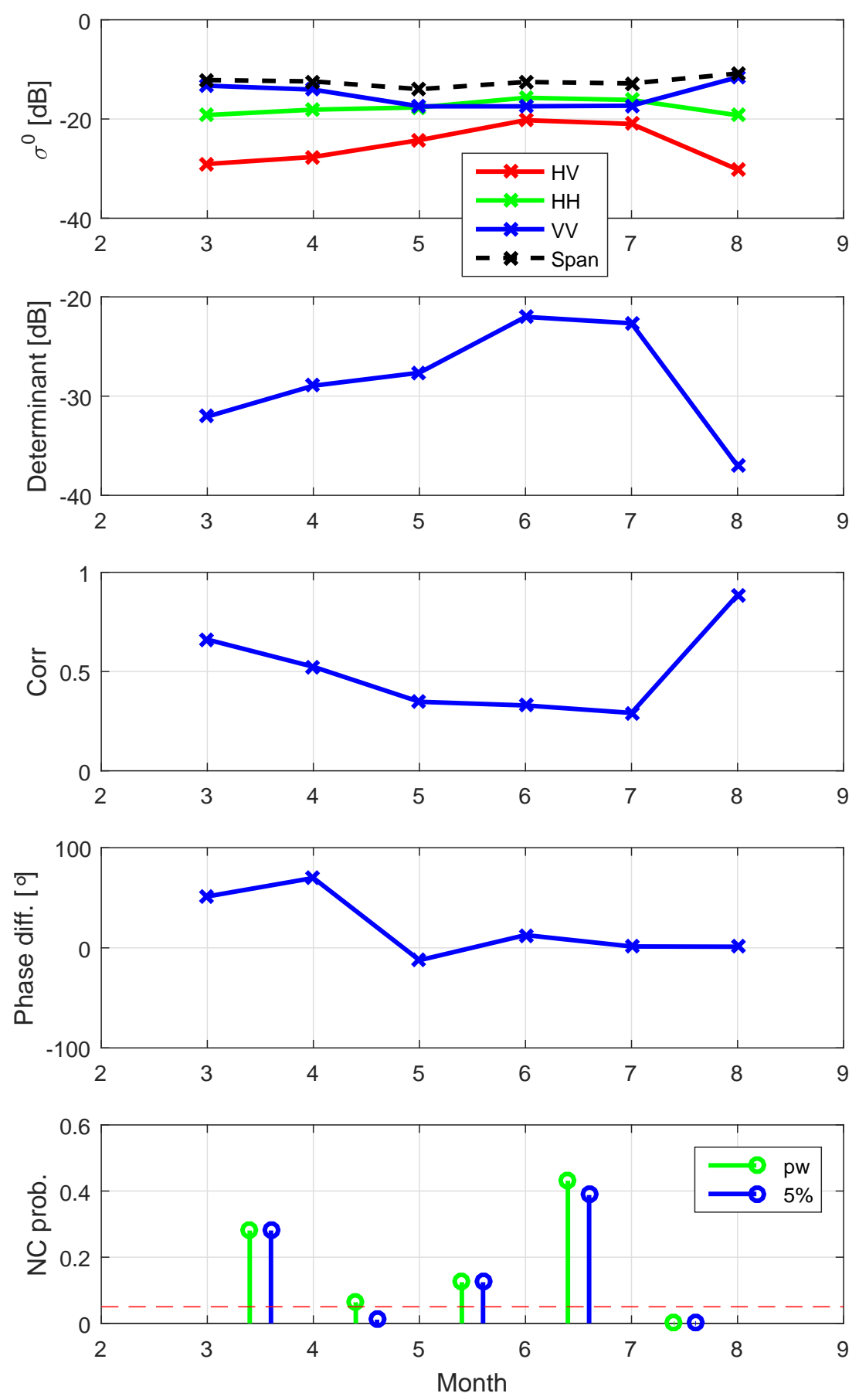

Figure 6: For a grass field this figure shows average (first plot) backscatter coefficients and span, (second plot) determinant of the covariance matrix multiplied by the equivalent number of looks, (third plot) magnitude of the correlation between $\mathrm{HH}$ and $\mathrm{VV}$, (fourth plot) phase difference between $\mathrm{HH}$ and $\mathrm{VV}$, and (fifth plot) pairwise (green stems) and omnibus test based no-change probabilities (at a $5 \%$ significance level, blue stems). 


\section{ACCEPTED MANUSCRIPT}

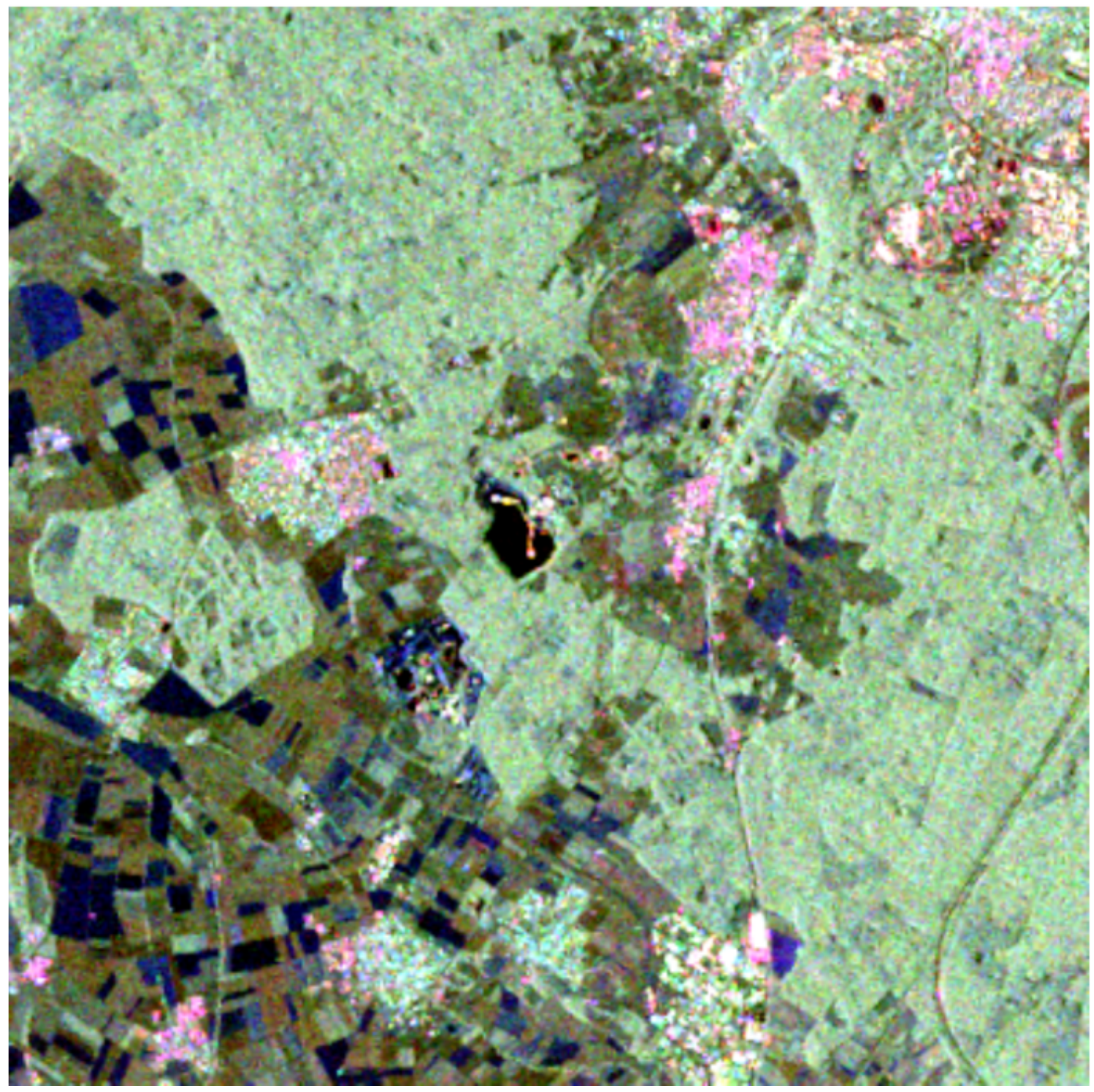

Figure 7: RGB color composite of a quad polarimetric RADARSAT-2 image acquired over an area southwest of Bonn, Germany on May 25, 2009, in the Pauli representation $\left(\mathrm{R}=\left\langle\left|S_{h h}-S_{v v}\right|^{2}\right\rangle\right.$ indicating double bounce, $\mathrm{G}=\left\langle\left|S_{h v}\right|^{2}\right\rangle$ indicating volume scattering, and $\mathrm{B}=\left\langle\left|S_{h h}+S_{v v}\right|^{2}\right\rangle$ indicating surface scattering or single bounce). RADARSAT-2 Data and Products (C)MacDonald, Dettwiler and Associates Ltd. (2009-2010) - All Rights Reserved. RADARSAT is an official trademark of the Canadian Space Agency. 


\section{ACCEPTED MANUSCRIPT}
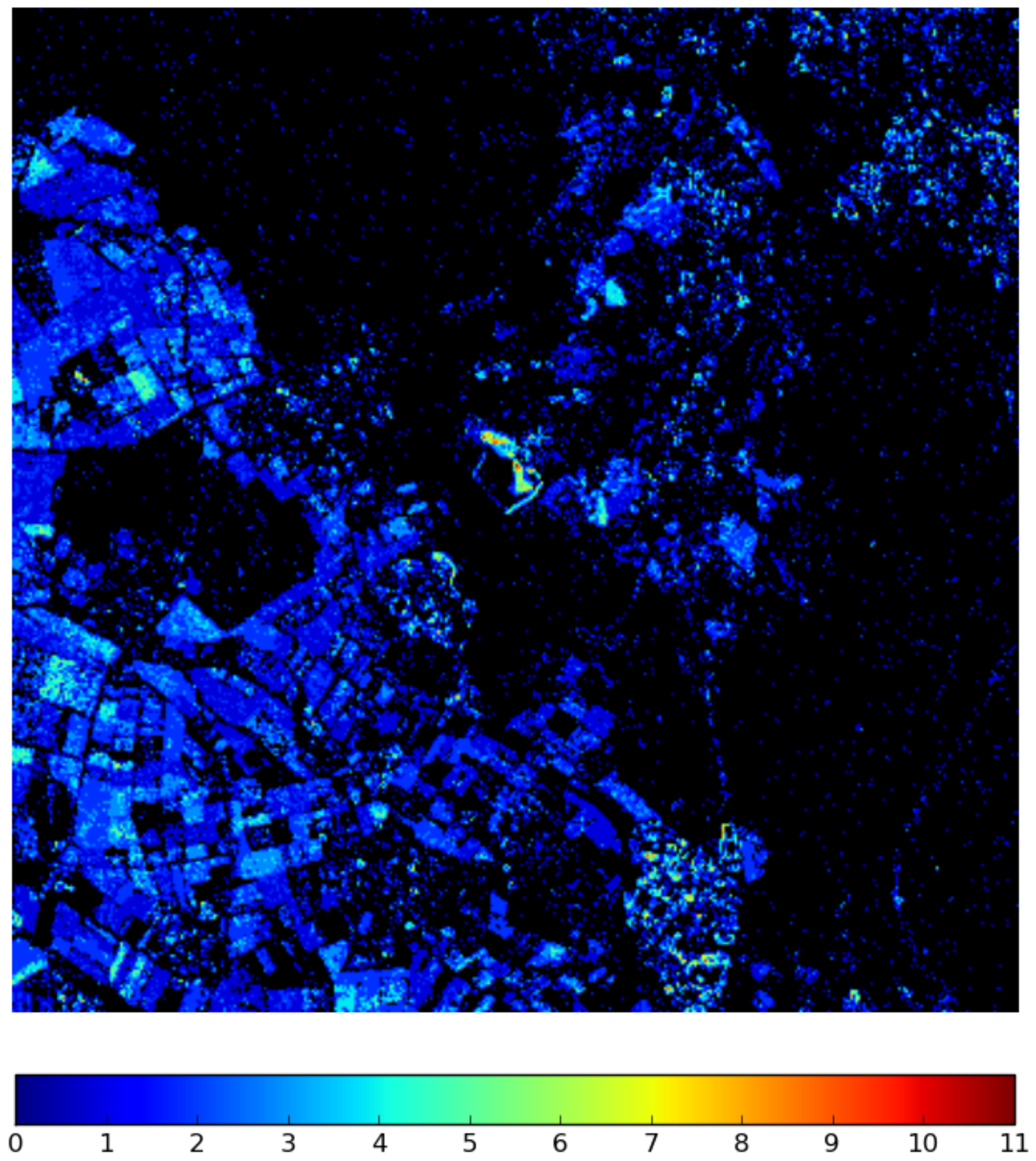

Figure 8: Change frequency map for a series of 12 RADARSAT-2 images acquired between May 25, 2009 and October 11, 2010 (same spatial subset as Figure 7). Change probability significance level is 99.99\%. RADARSAT-2 Data and Products (C)MacDonald, Dettwiler and Associates Ltd. (2009-2010) - All Rights Reserved. RADARSAT is an official trademark of the Canadian Space Agency. 


\section{ACCEPTED MANUSCRIPT}

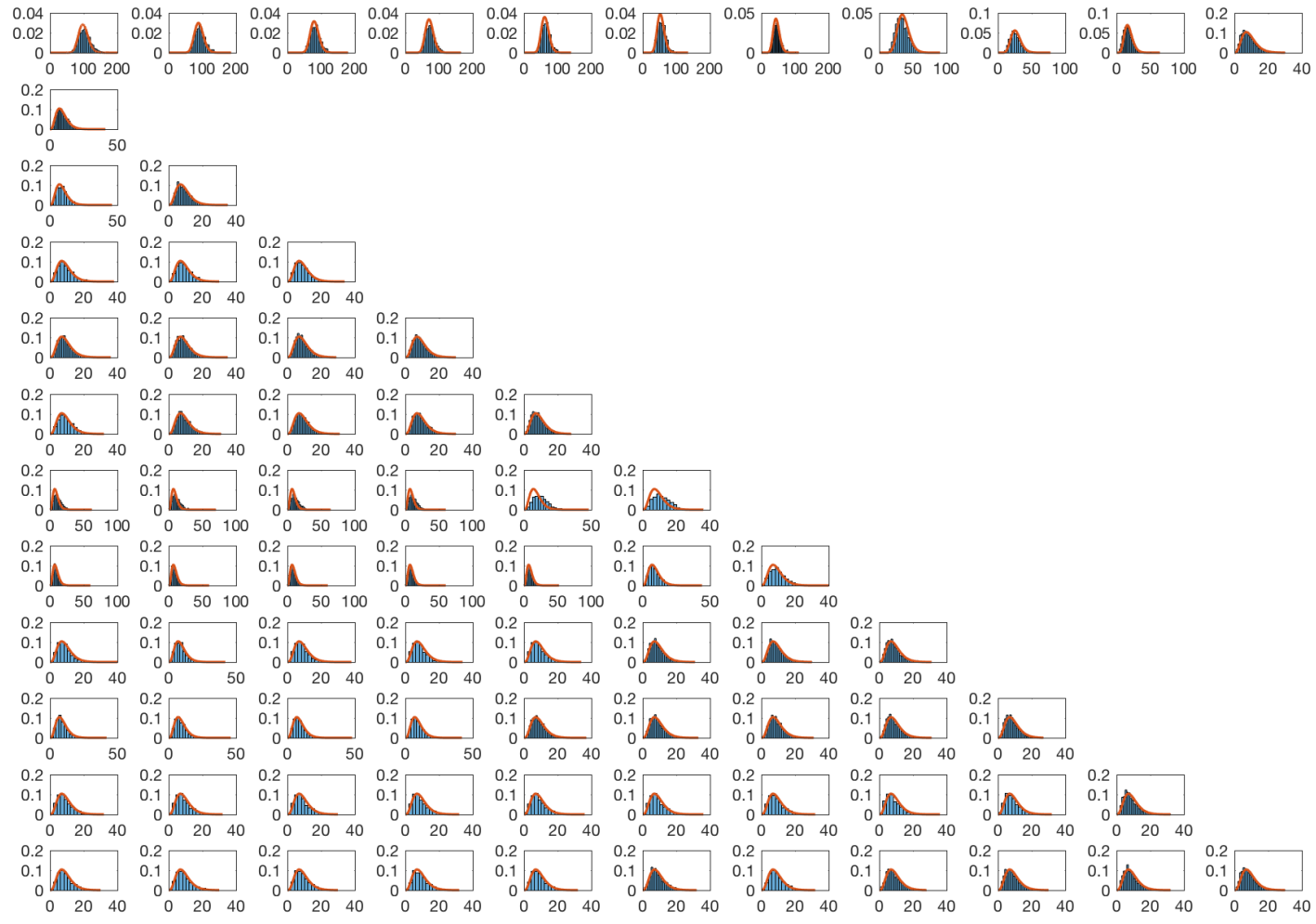

Figure 9: Histograms for an analysis of omnibus change for the RADARSAT-2 data along with the theoretical distributions for a no-change wooded area. For the $-2 \rho \ln Q$ (top row plots) the distributions are weighted sums of two $\chi^{2}$ distributions, see Conradsen et al. (2016). The numbers of degrees of freedom are 99 and 103, 90 and $94, \ldots$, 9 and 13, respectively. For all the $-2 \rho_{j} \ln R_{j}$ (the remaining rows) the distributions are again weighted sums of two $\chi^{2}$ distributions, the numbers of degrees of freedom are 9 and 13. Judged visually this illustrates a satisfactory fit between sample histograms and theoretical distributions for the test statistics in a no-change region. 